2013;436(3):530-535.

11. Rhodes JM, Simons M. The extracellular matrix and blood vessel formation: not just a scaffold J Cell Mol Med. 2007;11(2):176-205.

12. Moscatello DK, Santra M, Mann DM, McQuillan DJ, Wong AJ, Iozzo RV. Decorin suppresses tumor cell growth by activating the epidermal growth fac- tor receptor. J Clin Invest. 1998;101(2):406-412.

13. Goldoni S, et al. Decorin is a novel antagonistic ligand of the Met receptor. J Cell Biol. 2009; 185(4):743-754.

14. Schonherr E, Sunderkotter C, Iozzo RV, Schaefer L. Decorin, a novel player in the insulin-like growth factor system. J Biol Chem. 2005;280(16):15767-15772.
15. Carmeliet P, Jain RK. Molecular mechanisms and clinical applications of angiogenesis. Nature. 2011;473(7347):298-307.

16. Colin C, et al. Identification of genes differentially expressed in glioblastoma versus pilocytic astrocytoma using Suppression Subtractive Hybridization. Oncogene. 2006;25(19):2818-2826.

\title{
Aortic aneurysms in Loeys-Dietz syndrome - a tale of two pathways?
}

\author{
Frank Davis, Debra L. Rateri, and Alan Daugherty
}

Saha Cardiovascular Research Center, University of Kentucky, Lexington, Kentucky, USA.

Loeys-Dietz syndrome (LDS) is a connective tissue disorder that is characterized by skeletal abnormalities, craniofacial malformations, and a high predisposition for aortic aneurysm. In this issue of the JCI, Gallo et al. developed transgenic mouse strains harboring missense mutations in the genes encoding type I or II TGF- $\beta$ receptors. These mice exhibited several LDS-associated phenotypes. Despite being functionally defective, the mutated receptors enhanced TGF- $\beta$ signaling in vivo, inferred by detection of increased levels of phosphorylated Smad2. Aortic aneurysms in these LDS mice were ablated by treatment with the Ang II type 1 (AT1) receptor antagonist losartan. The results from this study will foster further interest into the potential therapeutic implications of AT1 receptor antagonists.

\section{TGF- $\beta$ and Ang II pathways in} thoracic aortic aneurysmal formation Aneurysms that present in the thoracic aorta have a wide range of syndromic and nonsyndromic associations (1). Marfan syndrome is one of the most researched syndromic associations and is attributed to a wide spectrum of mutations in fibrillin-1, which have been proposed to enhance the bioavailability of TGF- $\beta$ (2). The understanding of the role of TGF- $\beta$ in the etiology of thoracic aortic aneurysms (TAAs) moved forward with the development of mice that expressed the $\mathrm{C} 1039 \mathrm{G}$ mutant of fibrillin-1. These mice, colloquially referred to the "Marfan mouse," exhibit many Marfan-associated phenotypes, including a predisposition for aortic aneurysms. TGF- $\beta$-neutralizing antibody administration to Marfan mice prevented the characteristic media elastin disruption and aortic root expansion (3). Furthermore, the neutralizing antibody decreased canonical TGF- $\beta$ signaling in aortic smooth muscle cells, as defined by immunostaining of tissues for the phosphorylated form of Smad2

Conflict of interest: The authors have declared that no conflict of interest exists.

Citation for this article: J Clin Invest. 2014; 124(1):79-81. doi:10.1172/JCI73906.
(pSmad2). Another seminal discovery in the Marfan mouse was that administration of losartan, the initial member of the Ang II type 1 receptor (AT1R) blocker (ARB) class, ablated ascending aortic dilation. Subsequent studies in this mouse model have demonstrated that losartan-associated reductions in ascending aortic expansion are attributable to inhibition of the ERK pathway $(4,5)$. These groundbreaking studies in mice have assisted in development of multiple clinical trials that are evaluating efficacy of AT1R antagonism in thoracic aortic dilation of patients with Marfan syndrome (6). Although these studies shed light on the interactions between TGF- $\beta$ and AT1R signaling in TAA development, the specific mechanism of these interactions has not been elucidated (7).

TGF- $\beta$ was further implicated in the development of aortic aneurysms following the discovery of mutations in the genes encoding TGF- $\beta$ receptors in individuals afflicted with a clinical syndrome that has similarities to Marfan syndrome. This condition was subsequently termed Loeys-Dietz syndrome (LDS). Patients afflicted with LDS have a more aggressive form of ascending aortic dilation compared with those with Marfan syndrome (8). Dilation of the aortic root is detected very early, with documented aortic dissections occurring in patients with LDS as young as 3 months of age (9). Unlike Marfan syndrome, the vascular pathologies associated with LDS are more diffuse in location, as these aneurysms occur in other aortic regions and several vascular beds (10). The genetic basis of LDS is the presence of mutations in the genes encoding either type I or type II TGF- $\beta$ receptors (11). TGF- $\beta$ receptors function as multimers of both subtypes; therefore, clinical presentations are similar when defects are present in either receptor subtype. Although the TGF- $\beta$ receptor mutations result in impaired function, detection of enhanced Smad2 or Smad3 phosphorylation in surgical samples implies that TGF- $\beta$ signaling is actually increased in patients with LDS (11). The involvement of TGF- $\beta$ signaling in LDS development parallels the mechanisms of TAAs in Marfan syndrome. Unlike Marfan syndrome, there is a paucity of information on a role for Ang II in LDS.

\section{TGF- $\beta$ receptor mutations promote aortic aneurysms in LDS mouse models}

In this issue of the JCI, Gallo et al. (12) generated an array of mouse models with TGF- $\beta$ receptor dysfunction. These mouse models included mice with haploinsufficiency of either TGF- $\beta$ receptor (Tgfbr $1^{+/-}$ or Tgfbr2 ${ }^{+/-}$mice), knockin of LDS-associated alleles (Tgfbr $1^{M 318 R}$ or Tgfbr2 $2^{\text {G357W }}$ mice), and transgenic overexpression of the Tgfbr $2^{\text {G357W }}$ mutant. Haploinsufficiency of either receptor subtype did not produce vascular pathologies; however, heterogenous knockin of Tgfbr $1^{M 318 R}$ or Tgfbr ${ }^{\mathrm{G} 357 \mathrm{~W}}$ mutations or transgenic overexpression of mutated Tgfbr $2^{\text {G357W }}$ led to severe aortic pathologies. These included 


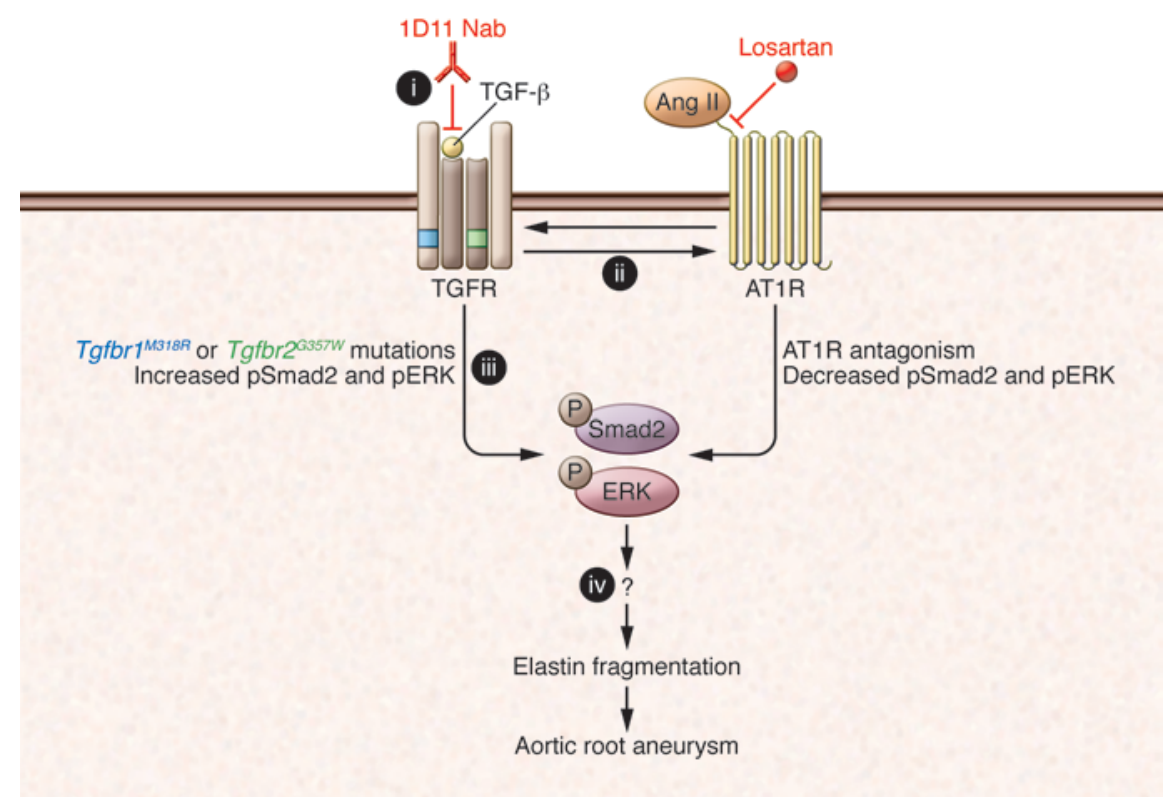

\section{Figure 1}

Convergence of the TGF- $\beta$ and Ang II signaling pathways is implicated in TAA. Missense mutations in either the TGF- $\beta$ receptor (TGFR) type 1 or 2 subtypes, specifically $\operatorname{Tgfbr}^{\mathrm{G}} 357 \mathrm{~W}$ and Tgfbr1M318R, lead to increased abundance of pSmad2 and pERK in mouse models of LDS. The increase in these activated proteins correlates with increased elastin fragmentation and aortic root dilation. Administration of losartan, an ARB, suppressed Smad2 and ERK phosphorylation and prevented aortic dilation. However, important mechanistic questions remain. (i) Why does administration of a TGF- $\beta$-neutralizing antibody (1D11 Nab) fail to reduce aortic root dilation? (ii) How does defective TGF- $\beta$ signaling augment AT1R stimulation? (iii) How do functionally defective TGF- $\beta$ receptors promote increased phosphorylation of Smad2 and ERK? (iv) What are the downstream targets of pSmad2 and pERK that cause elastin fragmentation and subsequent aortic aneurysm?

aortic root expansion and rupture and increased tortuosity of the thoracic aorta. Overall, mice expressing mutant forms of TGF- $\beta$ receptors exhibited many features of LDS, which permitted mechanistic studies of this disease.

Initially, mechanistic insight was gleaned from cultured smooth muscle cells harvested from aortic roots of wild-type, Tgfbr2 $2^{+/-}, T g f b r 2^{G 357 W}$ knockin, and transgenic mice. As expected, stimulation with TGF- $\beta$ promoted less $\mathrm{pSmad} 2$ abundance in cells expressing mutant receptors compared with controls. The lack of TGF- $\beta$ responsiveness was not attributable to differences in cell surface expression of the receptors. Vascular smooth muscle cells harvested from patients with LDS also demonstrated partial attenuation of TGF- $\beta$-induced Smad 2 phosphorylation. Receptor deficiencies did not lead to marked upregulation of TGF- $\beta$ secretion in mouse cells, and only TGF- $\beta 2$ secretion was increased in cells from patients with LDS. The results from these cell culture experiments corroborated previous studies that demonstrated decreased TGF- $\beta$ signaling as a result of LDS-associated TGF- $\beta$ receptor mutations (1).

Subsequent studies by Gallo et al. determined the fidelity of extrapolating these cell culture studies to the intact mouse (12). Western blot analysis of aortic root tissue from mice harboring either the Tgfbr $1^{M 318 R}$ or Tgfbr $2^{\mathrm{G} 357 \mathrm{~W}}$ mutation revealed that there was no alteration in pSmad2 abundance within the 24-week interval in which aortic expansion was measured. Given that analysis of whole tissue homogenates might obfuscate regional pSmad2 changes, Gallo et al. (12) performed immunostaining to visualize $\mathrm{pSmad} 2$ in aortic roots. While this technique does not easily lend itself to quantitative comparisons, greater immunofluorescent signals were observed in aortic media and adventitial tissues from $\mathrm{Tg} f b r 2^{\mathrm{G} 357 \mathrm{~W}}$ knockin mice compared with controls. The explanation as to why these data seemingly contradict the cell culture studies is certainly a matter of debate and intrigue. One potential issue is the promiscuity of Smad2 phosphorylation, which is not exclusively a result of TGF- $\beta$ signaling. Therefore, the use of Smad2 phosphorylation as an indicator of TGF- $\beta$ pathway activation could compromise data interpretation (13). Another intriguing aspect of the Gallo et al. study was that administration of a TGF- $\beta$-neutralizing antibody failed to prevent aortic root dilation, unlike the efficacy of this antibody treatment that has been demonstrated in the Marfan mouse (3).

In contrast to TGF- $\beta$ neutralization, Gallo et al. (12) provided convincing evidence that losartan administration ablated aortic pathology and expansion. Similar to the Marfan mouse, the decreased pathology was not mimicked by administration of a $\beta$-adrenoceptor antagonist (3). While losartan is primarily defined as an AT1R antagonist, its metabolites do have a potential range of other activities (14). Therefore, evaluation of other ARBs would assist in determining whether losartan-associated effects are due specifically to AT1R antagonism.

\section{Mechanism of TGF- $\beta$ and Ang II interactions in TAA development}

The authors conclude that aortic pathologies are generated by Ang II augmentation of TGF- $\beta$ signaling (Figure 1 ). Indeed, Ang II stimulates TGF- $\beta$ signaling by promoting secretion of TGF- $\beta$ isoforms from vascular smooth muscle cells (15). Conversely, TGF- $\beta$ signaling in vascular smooth muscle cells downregulates AT1R expression (16). The specific mechanisms by which defective TGF- $\beta$ receptors lead to augmented AT1R stimulation and generation of aortic pathologies in LDS is still a quandary. This issue is further complicated by evidence that suggests that TGF- $\beta$ promotes ascending aortic dilation through a combination of AT1R-dependent and -independent pathways (17). Clearly, additional studies are warranted to further elucidate the pathways that promote aortic aneurysm.

\section{Therapeutic implications}

Overall, the findings of Gallo et al. (12) provide important insight into the pathogenesis of aortic aneurysms in LDS. The authors demonstrated that a missense mutation in a single allele within either of the genes encoding TGF- $\beta$ receptor type 1 or 2 is sufficient to recapitulate LDS phenotypes in mice. In addition, these studies provide rationale for considering the 
application of AT1R antagonism as a therapy for patients with LDS. There is both retrospective and evolving prospective evidence that AT1R antagonism may be beneficial to patients with Marfan syndrome (6, $18,19)$. Together, the findings in patients with Marfan syndrome and the results from the Gallo et al. study (12) indicate that patients with LDS may potentially benefit from AT1R antagonism. Losartan has been the ARB of choice in most ongoing trials; however, the use of an ARB with a more favorable pharmacokinetic profile and longer half-life may enhance the protective effects against TAAs. The availability of the LDS mouse described by Gallo et al. (12) provides a model to determine the relative efficacies of this class of drugs before application to humans.

\section{Acknowledgments}

Frank Davis is supported by a Sarnoff Cardiovascular Foundation Fellowship. Research work is supported by funding from the NIH (HL062846 and HL107319).

Address correspondence to: Alan Daugherty, Saha Cardiovascular Research Center, Biomedical Biological Sciences Research Building, B243, University of Kentucky,
Lexington, Kentucky 40536-0509, USA.

Phone: 859.323.3512; Fax: 859.257.3235;

E-mail: Alan.Daugherty@uky.edu.

1. Lindsay ME, Dietz HC. Lessons on the pathogenesis of aneurysm from heritable conditions. Nature. 2011;473(7347):308-316.

2. Milewicz DM, Dietz HC, Miller DC. Treatment of aortic disease in patients with Marfan syndrome. Circulation. 2005;111(11):e150-e157.

3. Habashi JP, et al. Losartan, an AT1 antagonist, prevents aortic aneurysm in a mouse model of Marfan syndrome. Science. 2006;312(5770):117-121.

4. Habashi JP, et al. Angiotensin II type 2 receptor signaling attenuates aortic aneurysm in mice through ERK antagonism. Science. 2011;332(6027):361-365.

5 . Holm TM, et al. Noncanonical TGF- $\beta$ signaling contributes to aortic aneurysm progression in Marfan syndrome mice. Science. 2011;332(6027):358-361.

6. Moltzer E, Essers J, van Esch JH, Roos-Hesselink JW, Danser AH. The role of the renin-angiotensin system in thoracic aortic aneurysms: clinical implications. Pharmacol Ther. 2011;131(1):50-60.

7. Chen X, Lu H, Rateri DL, Cassis LA, Daugherty A. Conundrum of angiotensin II and TGF- $\beta$ interactions in aortic aneurysms. Curr Opin Pharmacol. 2013;13(2):180-185.

8. Loeys BL, et al. Aneurysm syndromes caused by mutations in the TGF- $\beta$ receptor. NEnglJ Med. 2006; 355(8):788-798.

9. Kalra VB, Gilbert JW, Malhotra A. Loeys-Dietz syndrome: cardiovascular, neuroradiological and musculoskeletal imaging findings. Pediatr Radiol. 2011;41(12):1495-1504

10. Gillis E, Van Laer L, Loeys BL. Genetics of thoracic aortic aneurysm: at the crossroad of transforming growth factor- $\beta$ signaling and vascular smooth muscle cell contractility. Circ Res. 2013;113(3):327-340.
11. Lindsay ME, et al. Loss-of-function mutations in TGFB2 cause a syndromic presentation of thoracic aortic aneurysm. Nat Genet. 2012;44(8):922-927.

12. Gallo EM, et al. Angiotensin II-dependent TGF- $\beta$ signaling contributes to Loeys-Dietz syndrome vascular pathogenesis. J Clin Invest. 2014; 124(1):448-460.

13. Rodríguez-Vita J, Sánchez-López E, Esteban V, Rupérez M, Egido J, Ruiz-Ortega M. Angiotensin II activates the Smad pathway in vascular smooth muscle cells by a transforming growth factor- $\beta$-independent mechanism. Circulation. 2005;111(19):2509-2517.

14. Sadoshima J. Novel AT(1) receptor-independent functions of losartan. Circ Res. 2002;90(7):754-756.

15. Subramanian V, Golledge J, Heywood EB, Bruemmer D, Daugherty A. Regulation of peroxisome proliferator-activated receptor-gamma by angiotensin II via transforming growth factor- $\beta 1$-activated p38 mitogen-activated protein kinase in aortic smooth muscle cells. Arterioscler Thromb Vasc Biol. 2012; 32(2):397-405.

16. Zhang XH, Zheng B, Gu C, Fu JR, Wen JK. TGF- $\beta 1$ downregulates AT1 receptor expression via PKC- $\delta$-mediated Sp1 dissociation from KLF4 and Smad-mediated PPAR- $\gamma$ association with KLF4. Arterioscler Thromb Vasc Biol. 2012;32(4):1015-1023.

17. Kuang SQ, et al. Aortic remodeling after transverse aortic constriction in mice is attenuated with AT1 receptor blockade. Arterioscler Thromb Vasc Biol. 2013; 33(9):2172-2179.

18. Brooke BS, Habashi JP, Judge DP, Patel N, Loeys B. Angiotensin II blockade and aortic-root dilation in Marfan's syndrome. N Engl J Med. 2008; $358(26): 2787-2795$.

19. Groenink M, et al. Losartan reduces aortic dilatation rate in adults with Marfan syndrome: a randomized controlled trial [published online ahead of print September 2, 2013]. Eur Heart J. doi:10.1093/eurheartj/eht334.

\title{
Toward postnatal reversal of ocular congenital malformations
}

\author{
José-Alain Sahel1,2,3,4,5,6,7 and Katia Marazova1,2,3
}

1INSERM, U968, Paris, France. 2Université Pierre et Marie Curie-Paris 6, UM80, Institut de la Vision, Paris, France. ${ }^{3}$ CNRS, UMR 7210, Paris, France. ${ }^{4}$ Centre Hospitalier National d'Ophtalmologie des Quinze-Vingts, INSERM-DHOS CIC 503, Paris, France. ${ }^{5}$ Fondation Ophtalmologique Adolphe de Rothschild, Paris, France. 6 Institute of Ophthalmology, University College of London, London, United Kingdom. ${ }^{7}$ French Academy of Sciences, Institut de France, Paris, France.

\begin{abstract}
Aniridia is a panocular disorder that severely affects vision in early life. Most cases are caused by dominantly inherited mutations or deletions of the PAX6 gene, which encodes a transcription factor that is essential for the development of the eye and the central nervous system. In this issue of the JCI, Gregory-Evans and colleagues demonstrate that early postnatal topical administration of an ataluren-based formulation reverses congenital malformations in the postnatal mouse eye, providing evidence that manipulation of PAX6 after birth may lead to corrective tissue remodeling. These findings offer hope that ataluren administration could be a therapeutic paradigm applicable to some major congenital eye defects.
\end{abstract}

Conflict of interest: José-Alain Sahel is a founder of and consultant for GenSight and Pixium Vision and a consultant for Sanofi and Gene Signal.

Citation for this article: $J$ Clin Invest. 2014; 124(1):81-84. doi:10.1172/JCI73560.
Mutations that inactivate gene function by promoting premature translational termination cause a large number of human diseases. It is thought that at least one-third of all genetic diseases and many types of can- cer are the result of such mutations $(1,2)$. These mutations are referred to as nonsense mutations, premature stop mutations, or premature termination codons (PTCs). Given that PTCs often result in a complete loss of protein function, the associated diseases usually manifest as severe phenotypes. Examples of PTC-associated diseases include CF, Duchenne muscular dystrophy (DMD), and aniridia, among others.

\section{Aniridia, a panocular disorder}

Aniridia is a rare eye disease with an estimated prevalence of approximately 1 in 40,000 to 1 in 100,000 individuals. It is present at birth and characterized by a total 